\title{
Effect of Ultrasonic Shot Peening on Microstructural Evolution of Duplex Stainless Steel S32750
}

\author{
Sijia Liu ${ }^{1}$ and Keesam Shin ${ }^{1}$ \\ 1. School of Nano \& Advanced Materials Engineering, Changwon National University, Changwon \\ 51140, Korea
}

Surface nanocrystallization is a highly efficient method to improve the performance of materials (i.e., corrosion resistance, fatigue resistance, wear resistance, etc.) [1]. Shot peening treatment, as one of the surface modification, has been widely applied in various materials. S32750 duplex stainless steel is usually used as tubing in the oil/gas delivery system, operated in corrosion environment under high pressure. During service, oil/gas induced pitting on tubing reduces the wall thickness and even causes the failure of the structure. Improving the corrosion resistance is a key for the application and maintenance of such materials.

In this work, Ultrasonic shot peening (USP) is applied to the duplex stainless steel (S32750) with the following objectives: 1) surface nanocrystallization of the materials, 2) comparison of the treatment processing parameters, 3) microstructural evolution and the mechanism upon shot peening process.

The S32750 stainless steel has been treated at $1070^{\circ} \mathrm{C}$ for $1 \mathrm{~h}$ to prepare the precipitation-free microstructure ( $\gamma$ and $\delta$ dual phase structure). Shot peening treatment was carried out on the heat treated specimen. Cross section electron backscattered diffraction (EBSD) analysis was used to distinguish the evolution of the dual phase structure. Specific depth transmission electron microscopy (TEM) analysis was applied to the characterization of the evolution of nanocrystallization. The hardness of the shot peened specimen was tested upon micro hardness test.

The microstructure was investigated at specific depth to understand the evolution and even the mechanism upon USP process. Changes in microhardness shows the hardness increased apparent in the USP treated specimen. Hardness increased at the top regions of the surface and then reduced to $\sim 250$ HV down to the depth at $\sim 300 \mu \mathrm{m}$.

Previous studies have explored the dislocation effect in deformed metals [2]. The TEM analysis showed that: (1) the original matrix structure consists of large grains (Fig. 1). (2) After the treatment, the dominant deformation structure in the $\gamma$-phase was twin. While the deformation structure in $\delta$-phase was high density dislocation and dense dislocation wall, whose arrangement leaded to the formation of subgrain boundary and final resulting in the refinement of original coarse grain. (3) Grain refinement was mainly observed in the $\gamma$-phase due to the formation of high fraction of the twin that divided the coarse grain into a finer size. 
References:

[1] K. Lu, Science (2014) 345.

[2] X. C. Liu, H. W. Zhang, and K. Lu, Science 342 (2013), 337.

[3] This work was supported by the National Research Foundation of Korea (NRF) grant funded by the Korean government (MEST) (No. 2011-0030058).
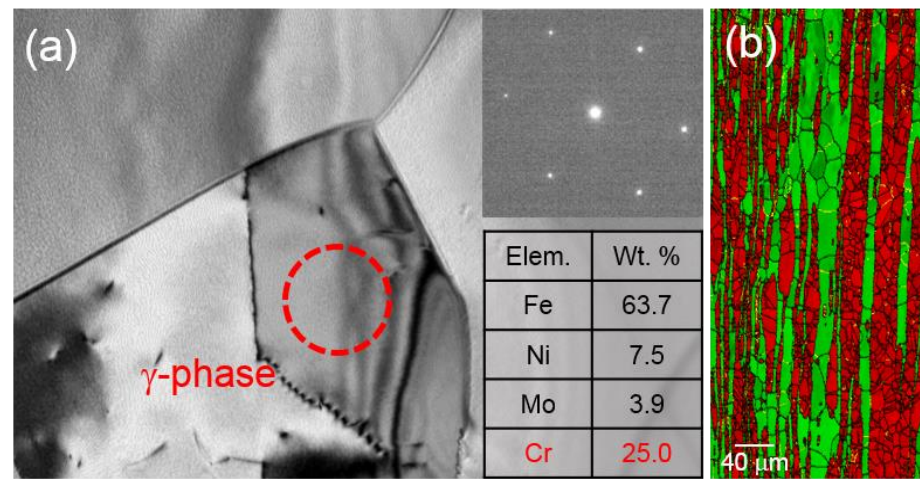

Figure 1. Typical (a) TEM image and (b) EBSD image of the untreated specimen.
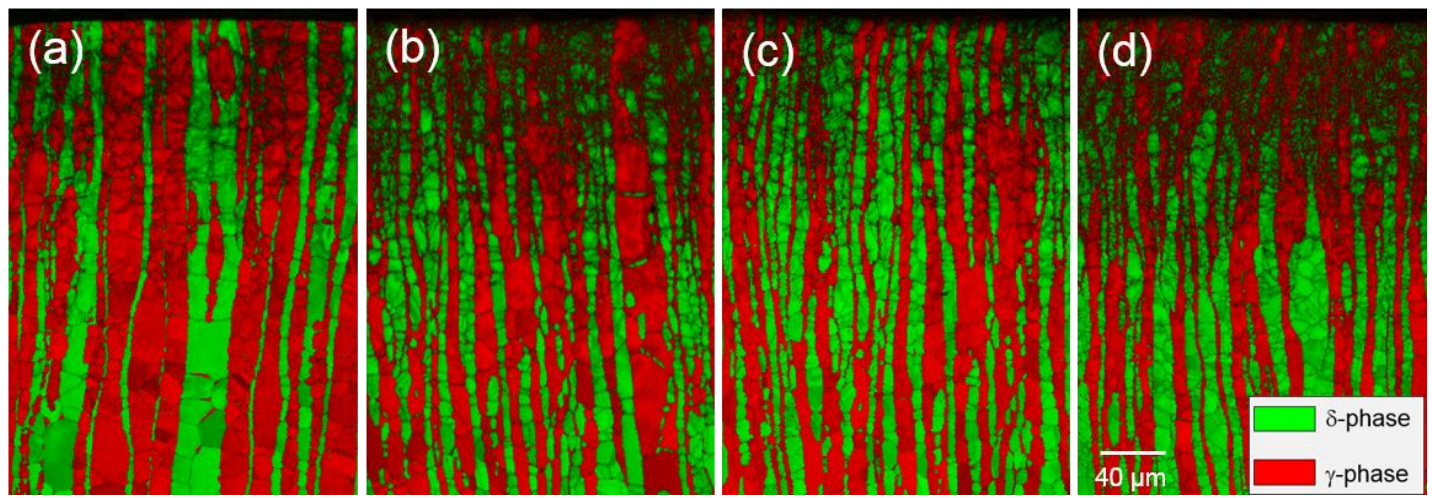

Figure 2. EBSD analysis of shot peened S32750 stainless steel, (a) $5 \mathrm{~min}$, (b) $10 \mathrm{~min}$, (c) $15 \mathrm{~min}$ and (d) $30 \mathrm{~min}$.
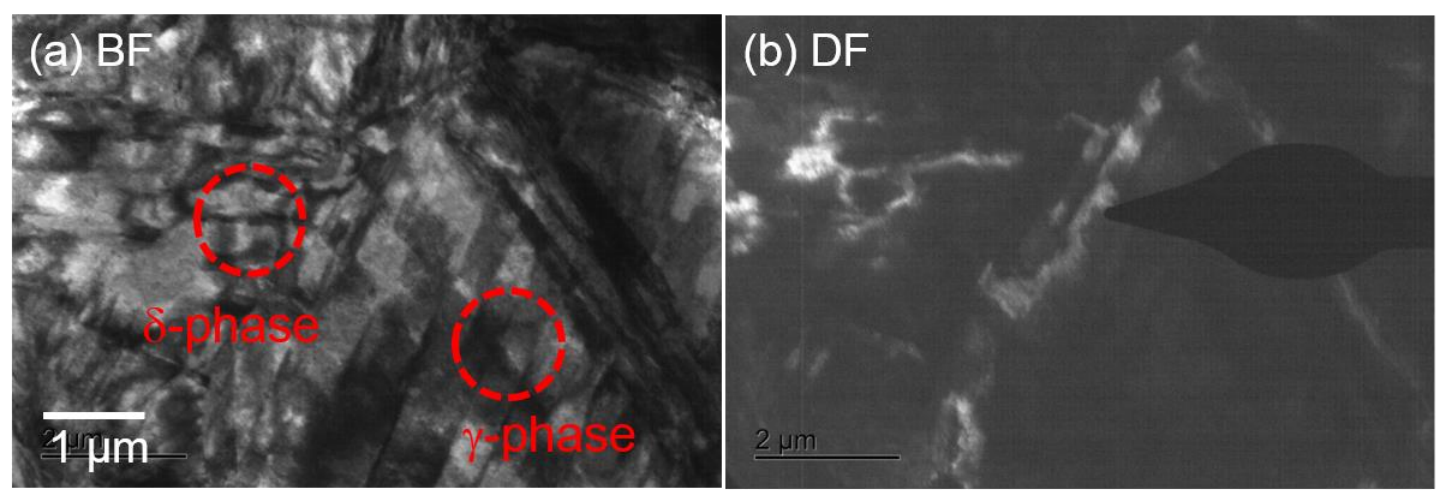

Figure 3. TEM analysis of USP-30min-top S32750 stainless steel, (a) bright field and (b) dark field. 